\title{
The adaptive nature of false memories is revealed by gist- based distortion of true memories
}

\author{
Timothy F. Brady ${ }^{1}$, Daniel L. Schacter ${ }^{2}$, George A. Alvarez ${ }^{2}$ \\ ${ }^{1}$ Department of Psychology \\ ${ }^{2}$ Department of Psychology \\ University of California, San Diego \\ Harvard University \\ San Diego, CA, 92093, USA \\ Cambridge, MA, 02138, USA
}

Please address correspondence to:

Timothy F. Brady

Department of Psychology

University of California, San Diego

9500 Gilman Dr. Dept. 0109

McGill Hall, Room 5322

San Diego, CA 92093

Email: timbrady@ucsd.edu 


\begin{abstract}
Human memory systems are subject to many imperfections, including memory distortions and the creation of false memories. Here, we demonstrate a case where memory distortion is adaptive, increasing the overall accuracy of memories. Participants viewed multiple real-world objects from a given category (10 airplanes, 10 backpacks...), and later recalled the color of each object. Participants were generally accurate, but even when they remembered having seen an item and remembered its color, they nevertheless reported the color as closer to the average color of its category than it really was. Although participants' memories were systematically distorted, they were distorted in a way that is consistent with minimizing their average error according to a simple Bayesian analysis. In addition, and consistent with the Bayesian analysis, the bias toward the category center was larger when participant's had greater uncertainty about the color of an item, but was present in all circumstances -- even when participants remembered an item, remembered its color, and reported high confidence in their color memory. Thus, memory distortion may not always be maladaptive: in some cases, distortion can result from a memory system that optimally combines information in the service of the broader goals of the person. Furthermore, this framework for thinking about memory distortion suggests that false memory can be thought of on a continuum with true memory: the greater uncertainty participants have about an individual item memory, the more they weight their gist memory; with no item information, they weight only their gist memory.
\end{abstract}

keywords: memory distortion; gist-based false memory; Bayesian model; visual long-term memory 
In addition to allowing us to remember objects and events that we have actually experienced, human memory systems are subject to distortions, biases, and the creation of false memories (Bartlett, 1932; Brainerd \& Reyna, 2005; Loftus, 2003; Schacter, 2001). Traditionally, memory distortions have been seen as failures of the memory system, with important theoretical and practical consequences (e.g., suggestibility of witnesses; Loftus, 1977, 2003). However, there are also potential benefits of our imperfect memory system: it has been proposed that apparent failings of memory may reflect the operation of adaptive properties that, in some way, enhance or even maximize performance across a range of tasks and situations (e.g., Anderson \& Milson, 1989; Bjork \& Bjork, 1988; Howe, 2011; Carpenter \& Schacter, 2017, 2018; Dewhurst, Anderson, Grace, \& van Esch, 2016; Newman \& Lindsay, 2009; Schacter, 2001, 2012; Schacter, Guerin, St. Jacques, 2011).

To understand how apparent memory imperfections can be adaptive, consider the important and well-studied phenomenon of gist-based or schema-based false memory, which occurs when people falsely recognize a word or picture that is conceptually or perceptually related to an item they saw previously. If participants see many images of boats, they are likely to falsely remember having seen a new image of a boat (Koutstaal \& Schacter, 1997); if participants are shown an office scene, they are likely to falsely remember seeing a stapler, even if one was not present (Brewer \& Treyens, 1981; Lampinen, Copeland, \& Neuschatz, 2001). Such gist-based representations do not lead only to false memories: gist-based representations can reflect retention of the essential meanings or themes of objects and events (e.g., Koutstaal, Verfaellie, \& Schacter, 2001), and can also allow us to prime related objects so we are quicker to recognize new objects when they are gist-consistent (e.g., Bar, 2004). Moreover, an individual's propensity for gist-based false recognition is correlated with their creativity (Dewhurst, Thorley, Hammond, \& Ormerod, 2011). Thus, properties of memory that at first seem to be imperfections may instead be adaptive for the broader goals of our memory system, such as recognizing the world around us quickly and accurately. 
One influential model of gist-based false memory appeals to two different kinds of memory representations: gist memories, summarizing information that was common across multiple items; and verbatim individual item memories, reflecting detailed information about a particular item (e.g., Brainerd \& Reyna, 2002, 2005; Reyna \& Brainerd, 1995; see also, Koutstaal \& Schacter, 1997). The basic idea is that when presented with an image that an individual actually did see - say, a particular boat people are likely to retrieve the item or verbatim trace and will accurately respond. When presented with an unrelated image, say a cow, people will not find a matching gist or verbatim representation, and will thus know that they did not see the item. However, when presented with a new boat that they did not see, they might retrieve the gist representation and believe they have seen the item before, resulting in a false memory (Brainerd \& Reyna, 1998, 2005; Koutstaal \& Schacter, 1997; Reyna \& Brainerd, 1995).

This approach typically assumes that when an individual item memory/verbatim trace is successfully recalled, the gist plays little role. For example, fuzzy trace theory explicitly makes the claim that when a verbatim representation is retrieved, the gist representation plays no role in participants' responses (as formalized by multinomial processing tree models; Brainerd, Reyna, \& Mojardin,1999; Brainerd \& Reyna, 1998; Erdfelder et al. 2009). However, within the context of an adaptive memory framework, this independence is unexpected: if people have access to a verbatim representation and a gist representation, then their responses could be more accurate if they combined these sources of information (e.g., Huttenlocher, Hedges \& Vevea, 2000; Brady \& Alvarez, 2011).

Combining item-specific representations and gist-based representations is useful in standard gistbased false memory situations because even item-specific information is not perfect (e.g., if asked to remember the color of an item, you might remember a greenish-yellow boat, but the boat could have been more green or more yellow). Thus, if you know something about what the items in a task were like in general (e.g., there were many green boats), then this gist information can inform your specific item 
memories (e.g., the boat you saw was probably on the greener side). Combining these sources of information will result in item representations that are more accurate on average, but that are also biased towards the gist of the set (Huttenlocher et al. 2000; Hemmer \& Steyvers, 2009; Brady \& Alvarez, 2011; see Appendix for a full derivation of how biases toward the gist increase accuracy). Thus, sometimes people should engage in systematic memory distortion: they should remember items as closer to the center of the category than they really were. Such a phenomenon may have been overlooked in models of gist-based false memory because in most paradigms using verbal stimuli or categorically distinct images, responses are digital: participants either remember the word or picture successfully or fail to do so. Here, the gist information does not provide any information that would influence responses above-and-beyond the verbatim, item-specific trace.

Recently, we have developed a continuous report method (Brady, Konkle, Gill, Oliva, \& Alvarez, 2013) to examine visual long-term memory in an analog fashion, enabling us to obtain continuous estimates of how well an item is remembered. In such a continuous report task, even verbatim representations are noisy: that is, if you are asked to remember the exact color of an item, you will not do so perfectly. Thus, gist representations - the color of the boats in general, for example -- have information that is helpful for the task, even when item-specific memory traces are available. Using this task, it is possible to measure distortion in item-specific memories and ask whether people are indeed combining gist and item-specific information, or whether these two memory traces are used entirely independently. 


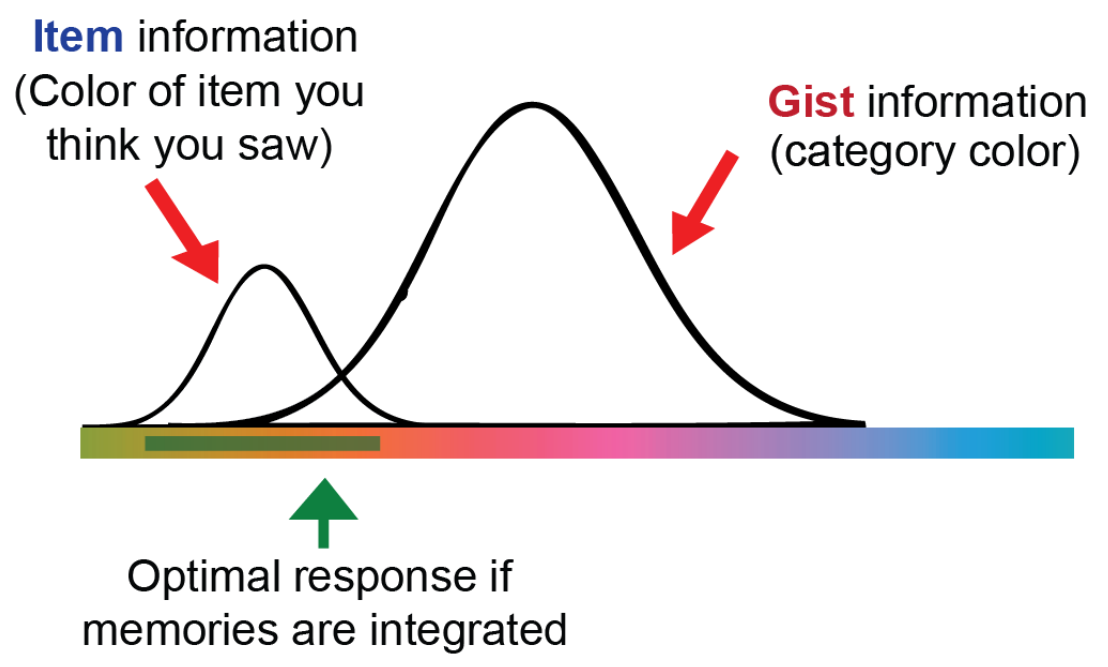

Figure 1. Schematic of the Bayesian integration model. If participants have uncertainty about the color of a particular item (left Gaussian; "item information"), and also know something about the color that items of the category were in general ("gist information"), they should combine these sources of information, resulting in a bias toward the category (optimal response; indicated by the green arrow). Intuitively, this is because it is more likely that this item was misremembered as more yellow than it actually was than it is that it was misremembered as more pink than it actually was, since most of the items of this category were near pink.

Why should integrating gist and item memories result in a systematic bias in memory for individual items? To understand this prediction, imagine you remember that a particular item was a certain color, $x$, but that you have some uncertainty about the exact color of the item due to both perceptual and memory noise (termed $\sigma$ ). If this is the only information you had available and you were asked what color the item was, your best answer would be to report $x$. However, if you have additional information about the item, then an optimal observer should make use of this information as well. For example, suppose you know that the items in the category were centered around color $\mu$ and had a certain spread, $\tau$. In this case, the optimal strategy to minimize your error is to report $(r)$ a weighted average of the category mean $(\mu)$ and $x$ (e.g., a weighted average of the information available in the gist and verbatim traces): 


$$
r=\frac{\tau^{2}}{\sigma^{2}+\tau^{2}} x+\frac{\sigma^{2}}{\sigma^{2}+\tau^{2}} \mu
$$

This is the Bayesian optimal response, and will produce the least error on average, but at the cost of producing a systematic bias towards the category mean (see Figure 1 for a schematic). This is true even when people have a fairly good idea what color an item was, although the more sure they are of the color (e.g., the smaller $\sigma$ is) the less they should weight the category mean. In general, a bias toward the category mean will result in less error than reporting purely the item-specific, verbatim trace you encode for a specific item (see Appendix).

Previous studies have shown that this kind of adaptive memory distortion occurs when participants have a pre-existing category or semantic memory and form a new episodic memory about an item from this category. For example, Hemmer and Steyvers (2009a) showed that participants integrate known semantic information about the size of fruits and vegetables with their episodic memory of the size of a particular item, showing biases to remember items' sizes as more like the prototypical sizes of their category (see also Feldman, Griffiths, \& Morgan, 2009; Hemmer \& Persaud, 2014; Hemmer \& Steyvers, 2009b; Huttenlocher, Hedges, \& Vevea, 2000). In addition, studies have shown that, when all the items in the entire study are drawn from a certain distribution, this distribution affects reproduction (Huttenlocher et al. 2000). These findings suggest that at least in some circumstances, participants make use of existing knowledge to influence their individual episodic memories in a rational way. However, it remains an open question whether this influence can also occur under conditions used to elicit gistbased false memories. In particular, when participants are presented a series of items from a variety of categories and must form new episodic memories for all of these items, do they combine the newlylearned episodic "gist" information (e.g., that all boats were greenish/yellow) with information about a particular individual item (that they remember a particular boat as a particular color)? If so, such a pattern would suggest that even episodically encoded gist information, which varies across items in the 
encoding session, as is typically present in gist-based false memory, can result in memory distortion (as opposed to existing category information, as in previous studies; Hemmer \& Persaud, 2014).

Thus, the current studies sought to examine the extent to which participants are biased toward the category's mean color in a continuous report task. The outcome of these studies should provide important information about how participants use their gist memories. If we observe no bias toward the category mean, this finding provides strong evidence that people make use of only an item-specific memory trace or a gist memory, but not both. If we observe a bias, this finding suggests that participants integrate item-specific information and gist information to arrive at a more accurate response, a kind of memory distortion that is in line with what we would expect from an adaptive perspective on false and true memory, where memories are distorted in the service of the broader goals of the memory system (e.g., minimizing error).

\section{EXPERIMENT 1}

We conducted two experiments to test the prediction that participants would be biased toward the category mean in reporting the color of items, including items that they successfully remember. In Experiment 1, participants were shown 40 items from a mixture of 4 different categories before being tested on those item's colors. Remembering the color of 40 different items should be challenging and thus lead to noisy item representations (e.g., uncertainty about the exact color of any given item). According to the Bayesian integration model, noisy item representations should lead to reliance on the gist of the category, and thus result in a bias toward the category mean. 


\section{Method}

\section{Participants}

24 participants from the Harvard University or Cambridge, MA community were tested (13 female; age range 18-25). All participants gave informed consent and had normal color vision, as assessed using Ishihara's test for color deficiencies (1936).

\section{Apparatus}

Experiments were run in MATLAB using the Psychophysics Toolbox (Brainard, 1997; Pelli, 1997), with stimuli presented on a $49^{\circ}$ by $31^{\circ}$ display viewed from approximately $57 \mathrm{~cm}$.

\section{Stimuli}

Stimuli consisted of 300 objects on white backgrounds (200 for study, 100 as foils). The objects came from 20 distinct categories (e.g., airplanes, cellphones, lamps, backpacks), with 15 objects per category. Each object was chosen so that it consisted largely of a single arbitrary color and so that the object would be recognizable in any color (see Figure 2). This procedure allowed us to rotate the hue of the objects so they could appear in different colors (as in Brady et al. 2013). We treated hue as a circular dimension, with hues ranging from $0^{\circ}$ to $360^{\circ}$, such that $0^{\circ}$ corresponded to red hues and $180^{\circ}$ to green hues. 


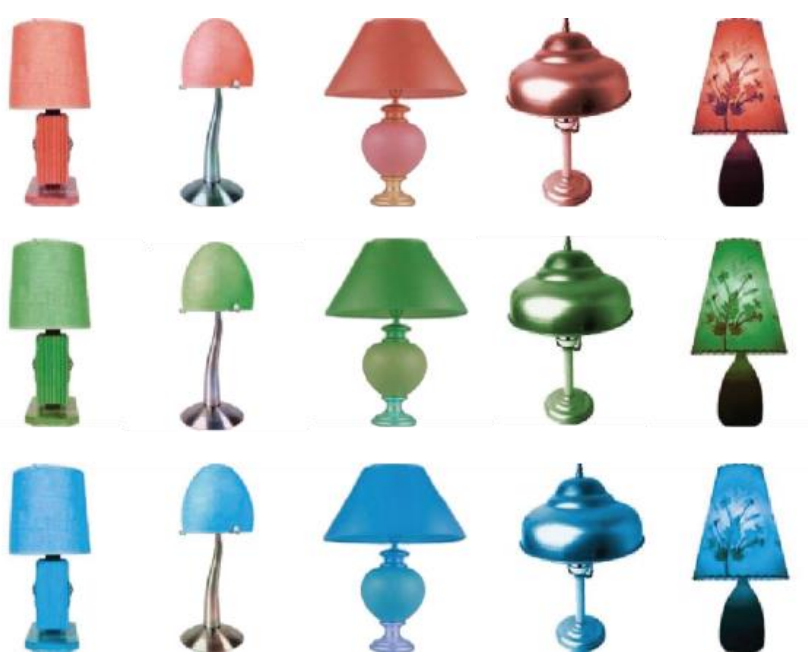

Figure 2. Example stimuli. 5 of the 15 lamps, each shown in 3 colors.

To change the color of a stimulus, each pixel was independently rotated through an equiluminant circle in $\mathrm{L}^{*} \mathrm{a}^{*} \mathrm{~b}^{*}$ space. Stimuli were modified so their colors fell on approximately the same color circle used by Zhang and Luck (2008). Throughout the experiment, all stimuli were presented at the center of the screen and subtended $\sim 6^{\circ}$ of visual angle.

\section{Procedure}

Participants were presented with a sequence of objects and asked to remember both a) which objects they saw and b) the exact color in which those objects were presented. Stimuli were presented in 5 blocks of 40 objects each. Within a block, objects were presented one at a time for 2 seconds each, and after each block, participants were tested on their memory for the objects from that block. Each block consisted of 10 objects from each of 4 different categories (e.g., 10 airplanes, 10 cellphones, 10 lamps, 10 backpacks; Figure 3A). Images from the same category were all shown in similar colors, so that each category had a particular color "gist" (e.g., all bells might be blue/green for one participant). To achieve this, each of the categories was assigned a random color to function as the central color of that category $(\mu)$, and then the colors for the particular 10 items were randomly sampled from a von Mises 
distribution (Fisher, 1993) with mean $\mu$ and the concentration parameter ( $\mathrm{k}$ ) set to 4 . The von Mises distribution is a circular analog of the normal distribution, and a concentration ( $\mathrm{k}$ ) of 4 corresponds to a standard deviation of approximately $30^{\circ}$ (see Figure $4 \mathrm{~A}$ for a visualization of the breadth of this distribution). Thus, for one participant, backpacks might be orange/pink/red/purple, whereas for another they might be green/teal/blue/purple, etc; with each of the individual items having a color randomly sampled from this color set (see Figure 1).

\section{(A) Study}

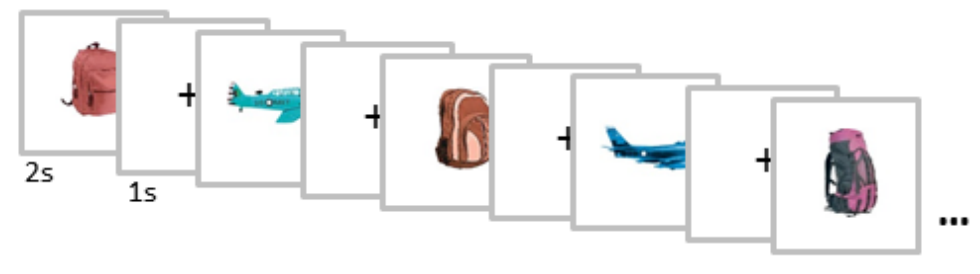

(B) Test

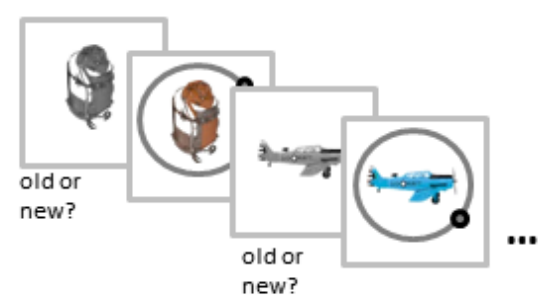

Figure 3. (A) Structure of the study period. In each study block, participants saw 10 objects from each of 4 different categories (40 objects total), with the objects from a given category appearing in a similar color. Participants were instructed to remember both the objects and their colors. (B) Structure of the test period. Participants were presented with 20 objects they previously studied and 20 they hadn't, and for each object were required to give an old/new judgment as well as to report the precise color of the object using a color wheel.

After studying the 40 images from a given block, participants were given 40 test trials. 20 of these assessed memory for old items ( 5 from each category), and 20 of them presented foil items ( 5 from each category). We assessed memory for only half of the studied items because each color report trial took 10 seconds, and thus the set of 40 test trials was fairly long. On each test trial, a test item was initially presented on the screen in grayscale, and participants had to indicate whether this item was old or new using the keyboard. Then, regardless of whether they reported that an item was old or new, participants were asked to report the color in which the item had been presented. During the instructions period, we 
explained that memory is fallible and that even if participants thought they had not seen an item, we still wished for them to provide their best guess for what color it might have been.

Participants' color memory was measured using the method of adjustment (see Figure 3B). At the beginning of the color memory test, the item remained in grayscale and the mouse pointer appeared at the center of the item. When the participant moved the mouse, the test item appeared in color. The angle between the mouse and the center of the test item determined the item's hue, and a dot was presented along the adjustment ring indicating the current position. When participants decided that the current color was correct, they clicked the mouse. The angular error between the correct color and the clicked color was taken as a measure of the accuracy. The color wheel was randomly rotated across trials so that the spatial position of the mouse did not correspond directly to a particular color.

Participants proceeded at their own pace and were asked to be as accurate as possible in their decisions. Before beginning, participants were given several kinds of practice trials. The first 4 practice trials consisted of simply remembering 1 item and then reporting its color. The next 4 consisted of remembering 2 items per trial and then responding to a test item, which they had to indicate was either old or new, and then indicate its color. The final practice trial consisted of a set of 10 objects from the same category; participants had to remember all 10 objects and then perform the old/new task and color task.

\section{Results}

Participants performed well on the old/new task, with an overall hit rate of $72.4 \%$ (standard error (SEM): $\pm 2.0 \%$ ), and false alarm rate of $26.7 \%$ (SEM: $\pm 2.6 \%$ ). Thus, participants were largely successful in distinguishing old from new items ( $d^{\prime}=1.3$; SEM: \pm 0.09 ). 
To address our hypothesis that participants would integrate gist information with their itemspecific memories, we examined the data from the color report task. Looking only at hits (e.g., items participants correctly reported seeing) ${ }^{1}$, we found that participants were fairly accurate in reporting the color of items (average error: $37.7^{\circ}, \mathrm{SEM}: \pm 2.0^{\circ}$ ). Random guessing would result in an error of $90^{\circ}$, and participants performed at a significantly more accurate level than this baseline $(t(23)=26.5, p<0.0001)$. However, participants were biased toward the color of the category even on these trials where they reported remembering the items. To demonstrate this point, we calculated participants' error on each item, aligned such that positive errors were in the direction of the category center and negative errors were away from the category center (see Figure 4B). Thus, more accurate responses are closer to 0 , and errors toward the category are on the positive side of the plot. On average, participants reported a color $25.9^{\circ}$ (SEM: $\pm 3.3^{\circ}$ ) from the correct answer in the direction of the category center, a significant bias (different from $0^{\circ} ; t(23)=7.86, p<0.0001$ ). This bias was highly reliable, with 23 out of 24 participants showing a bias in the predicted direction.

It is not clear whether this bias arises from integration of the gist and item-based memory traces at encoding, during maintenance or at retrieval. However, participants' responses tend to be biased toward the category mean even for the first item they saw from a particular category during the encoding phase (mean: $23.0^{\circ} ; \mathrm{SEM}: \pm 11.2^{\circ} ; t(23)=2.05, p=0.052$ ), so it is not necessary for participants to have known the category's mean color at the time of encoding the item to show a bias. This finding suggests that the bias does not arise from a distorted encoding the color of the item when it is initially presented (e.g., as closer to the category center than it really is).

\footnotetext{
${ }^{1}$ Participants did not show any evidence of using item-specific color information on 'miss' trials; responses on such trials were a mixture of totally random responses and responses reflecting only the gist of the category the item was from.
} 

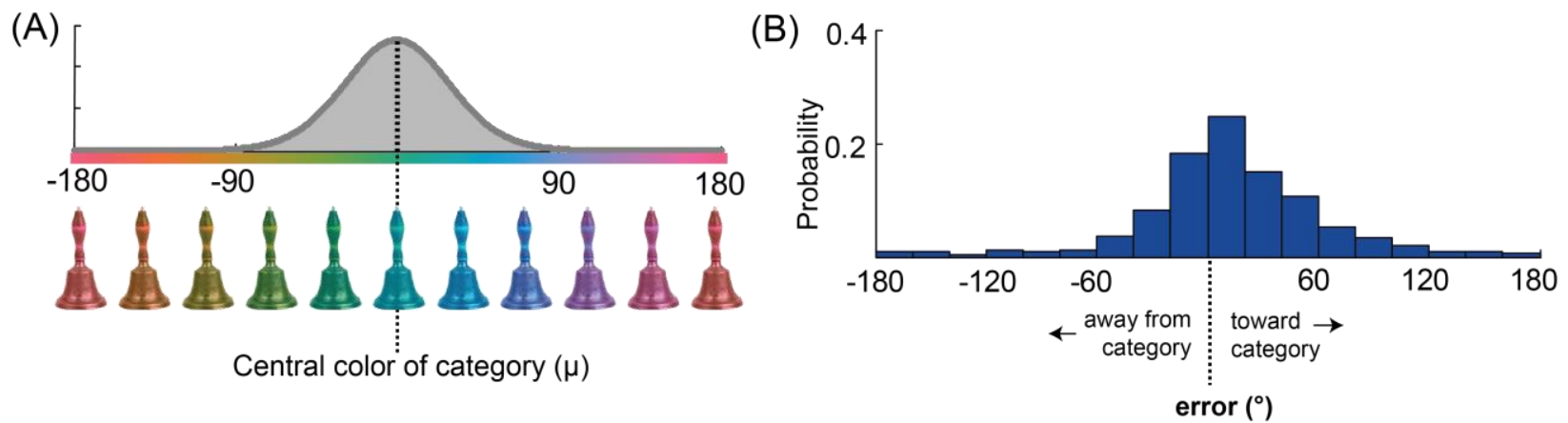

Figure 4. (A) The color distribution for items within a category. All the items in a given category were shown in similar colors; in particular, their colors were sampled from a von Mises distribution with $\mathrm{k}=4$ (shown). Thus, if the objects from the category "bell" were centered on turquoise, items from that category would almost never be yellow but might be anywhere in the green or blue hues. (B) Color report analysis, showing error from the correct answer, with positive errors reflecting errors in the direction of the center color of the category. Participants were biased to report items towards the central color of the category. Data shown here are collapsed across all participants, but all statistics are reported based on individual participants.

The presence of a bias towards the category center does not necessarily indicate that gistinformation and item information are integrated. Instead, participants could show such a bias as a result of a "mixed" strategy, rather than integration. In particular, it is possible that participants' responses represent a mixture of two kinds of trials: on some trials, participants might remember the color and report it accurately (giving rise to a normal distribution centered around $0^{\circ}$ error); on other trials, participants might not have known the answer at all, and so they might use only the gist and report a color that is consistent with the category of the item (e.g., blue-green if the item was a bell). On this account, the distributions we observe in Figure 4B would result from the sum of two separate distributions: one for remembered trials, and one for gist-only trials. While gist-only trials would necessarily be biased toward the category mean, remembered trials would not be.

It is possible to distinguish this account from the integration account by making use of the trials where participants commit false alarms to items that were not present in the study stream. In particular, the colors that participants report on false alarm trials allow us to estimate the colors they would report 
using gist-only information (i.e., a gist-only distribution), since these are trials where participants did not know anything about the color of the particular item (never having seen it) but did have information about the category of the item and the color distribution of this category. Thus, we can estimate what participants' error distribution would look like if they used this same information (reporting only based on the category, not the item information) on some proportion of the "old" trials. To simulate this distribution, we took the colors that participants reported on each false alarm trial, and, for each such trial, calculated how far the reported color was from the color of each of the 'old' items in the same category. This procedure gives us an estimate of the entire distribution of errors we would expect to observe on old items if participants used only category information to respond to them, with no itemspecific information present.

The "mixture" account predicts that the color distribution on "hit" trials consists of this gist-only distribution plus some remainder that represents item-specific information. Furthermore, the mixture account predicts that this remainder, representing item-specific information, is symmetric and is not biased toward the category's mean color (i.e., is centered around zero). These predictions are not consistent with the data. In particular, no matter what proportion of hit trials we assume are actually gist-only response trials, the residual (e.g., the trials with item-specific information present) always shows a bias toward the mean color of the category (see Figure 5B and 5C). To show this bias, we binned response errors in 20 deg. increments between -180 and 180 deg. For any given proportion of gist-only trials, we computed the expected gist-only distribution, and subtracted that gist-only distribution from the observed distribution for hit trials. The critical question is whether the residual, reflecting itemspecific memory, still shows a bias towards the category.

For this analysis we exclusively considered gist-only response proportions between 1 and 61\%, since we can statistically reject the idea that more than $61 \%$ of the color responses on old trials arise 
from gist-only responding: above $61 \%$, the predicted gist-only distribution is significantly greater than the observed "old" distribution in at least one bin $(p<0.05)$, which is incompatible with the idea that the "old" distribution consists of gist-only responses plus other item-specific responses ${ }^{2}$. In other words, if you examine Figure 5B, the hypothesis of a 'mixture' account is that the distribution of colors for "old" items (blue line) is made up of both some proportion of trials representing gist-only responses (red line), plus some other responses reflecting responses with item-specific information (the difference between red and blue). Figure 5B shows the distributions if we assume gist-only responses make up $60 \%$ of the "old" distribution; near the maximum possible. We can see that this is the maximum because if we instead assumed that 70 or $80 \%$ of the responses were gist-only, the red distribution would be 10 or $20 \%$ higher everywhere, and thus the red distribution representing gist-only responses would be higher than the blue, "old" distribution, in several bins. This hypothetical scenario would require a negative proportion of item-specific responses, which is incoherent. In other words, if the red gist-only distribution is greater than the blue "old" distribution at any point, this arrangement is incompatible with the idea that old responses (in blue) are made up of both gist-only responses (red) plus itemspecific responses.

At all values of the gist-only response rate between 1 and 61\%, the residual (the difference between the true distribution and the computed gist-only distribution), representing the item-specific information, continues to be biased toward the mean color of the category, and at, all values, this bias remains significantly above zero (Figure 5A; gray shading represents the standard error of the mean).

\footnotetext{
${ }^{2}$ While we cannot reject the idea that up to $60 \%+$ of participants' responses could potentially be based on gistonly information, this represents an upper bound: this analysis demonstrates the item-specific component of participants' responses is biased even when assuming as much of the distribution as possible arose from gist-only responses. It is likely that not all $60 \%$ of these possible gist-only responses really do represent purely gist-only information, especially since gist-based false memory tends to be less common with visual images (e.g., pictures), since visual images evoke distinctive memory traces (Schacter, Israel, \& Racine, 1999) and are well remembered when appropriately tested (Brady, Konkle, Alvarez \& Oliva, 2008; Guerin, Gilmore, Robbins, \& Schacter, 2012).
} 

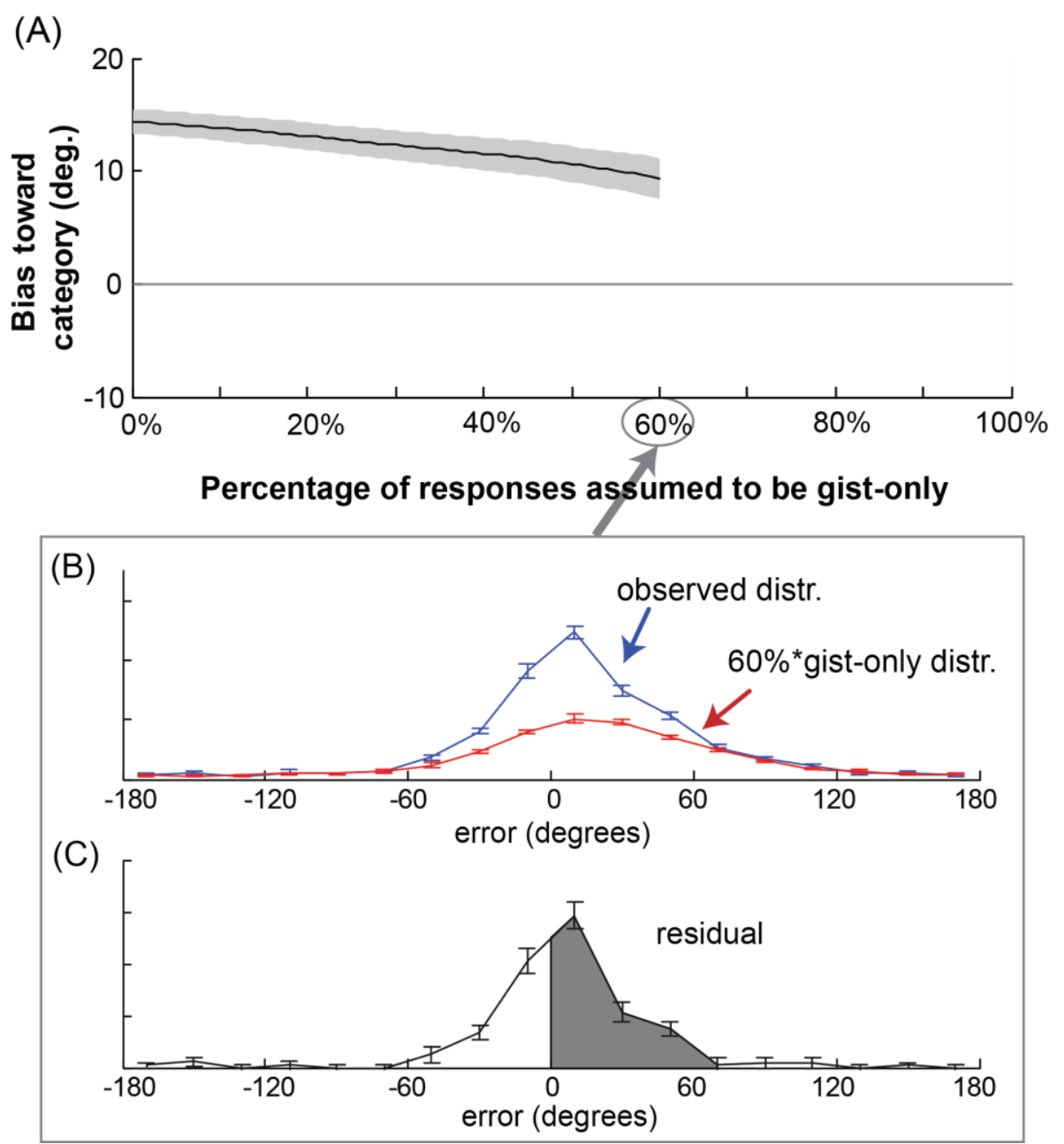

Figure 5. (A) The remaining bias toward the category mean after removing the gist-only distribution. No matter what percentage of the responses are assumed to be gist-only responses ( $x$-axis), the itemspecific information in the residual continues to be biased toward the category's mean color (greater than 0 degrees on the $y$-axis). The shaded region represents the standard error of the mean across participants. (B) How we calculate the bias at a given rate of gist-only responses, focused on the point where $60 \%$ of the responses are assumed to be gist-only. The blue line shows participants' actual error distribution, as in Figure 4B. The red line shows the calculated error distribution from participants' false alarms, also flipped to have positive be toward the category mean, and multiplied by 0.60 to simulate $60 \%$ of items being gist-only; error bars represent the standard error of the mean. (C) The residual - the blue error distribution minus the red gist-only distribution - shows what the distribution of responses looks like after factoring out gist-only responses. Notice that a majority of responses are still toward the category's mean color ( $>0$; shaded). 
Thus, even if some proportion of participants' responses do reflect cases where participants had no item information and responded only based on category information, the remainder of the responses do not reflect unbiased reports of the color. Instead, participants appear to be systematically biased toward the mean color of the category even in the remaining trials where item-specific information was used. Thus, we find significant evidence in favor of an account where participants combine gist and item-specific information, resulting in a bias toward the category mean even for remembered objects.

\section{Discussion}

Even when people remember having seen an item and remember the item's color, they nevertheless are systematically biased to report its color as closer to the mean color of its category than it was. This is true even after removing any proportion of trials that might reflect purely gist-only information (without any item information). Thus, this bias reflects an integration of item-specific information with gist information. This finding is consistent with the optimal Bayesian strategy: reporting a biased color reflects that participants used not only item-specific information but also category-level information to help them respond accurately, which results in less error on average than relying on only item-specific memories.

The results from this experiment suggest that participants are biased toward the central color of the category in a relatively difficult color memory task. However, the Bayesian model predicts that this bias should scale with the precision of participants' item memories: when participants have greater uncertainty about the color of an individual item, they should rely more on the gist; when they have more certainty, they should rely less on the gist. In particular, in the formula for deriving the optimal response, as the standard deviation of an individual item memory $(\sigma)$ increases, reflecting less precision, 
the weight on the item-specific memory $(x)$ goes down, whereas the weight on the category mean $(\mu)$ increases:

$$
r=\frac{\tau^{2}}{\sigma^{2}+\tau^{2}} x+\frac{\sigma^{2}}{\sigma^{2}+\tau^{2}} \mu
$$

This weighting reflects the fact that, if you know very precisely the color of a particular item, you should be almost unaffected by the gist; whereas if you have a lot of uncertainty about the color of a particular item, you should rely more on the information you have about what items of that category were like in general.

To test this prediction, in Experiment 2 participants were shown only 10 items (all from the same category) before being tested on those items' colors. In addition, participants were asked how confident they were in their color memory. This arrangement allows us to examine whether trials with more precise item memories lead to less bias toward the mean of the category. In addition, it allows us to ask whether there is still a bias toward the mean color of the category even for items remembered the most precisely. Finding a bias toward the category center even when participants accurately report the color with high confidence would suggest that this bias is a general property of memory.

\section{EXPERIMENT 2}

\section{Method}

\section{Participants}

To break down participants' responses by confidence-level, we needed substantially more data than in Experiment 1. Because we have a limited number of images that can be color-rotated, we could not easily increase the number of trials per participant. So we instead decided to recruit using Amazon's Mechanical Turk. We ran 200 participants from the United States; all participants were $18-55$ years 
old ${ }^{3}$, and gave informed consent in accordance with the procedures and protocols approved by the Harvard Committee on the Use of Human Subjects. Turk users form a representative subset of adults in the United States (Berinsky, Huber, \& Lenz, 2012; Buhrmester, Kwang, \& Gosling, 2011), and data from Turk are known to closely match data from the lab on memory tasks (Brady \& Alvarez, 2011; Brady \& Tenenbaum, 2013). All participants gave informed consent and reported that they had normal or corrected-to-normal color vision.

An additional 2 participants were run on the task but were replaced because they failed to discriminate old items from new items in the old/new task $\left(d^{\prime} \leq 0\right)$.

\section{Procedure}

The experiment was broken up into 10 blocks. As in Experiment 1, each block consisted of a study phase and a test phase. However, in this experiment, the study phase for each block was shorter, consisting of only 10 objects, all from the same category. During the study phase, objects were presented one at a time for 1 second each. As in Experiment 1, all 10 images from the category were shown in similar colors, sampled from a von Mises distribution with $\mathrm{k}=4$, so that each category had a particular color "gist".

After studying the 10 images from a given block, participants were given 10 test trials. 5 of these assessed memory for old items, and 5 of them were foil items from the same category. The test trials were identical to Experiment 1 except that after reporting the color, participants were asked to assess their confidence in their color memory on a 1-6 scale ("totally guessing" through "extremely sure"). In addition, unlike in Experiment 1, we did not ask participants to report the color of an item when they reported that the item was new.

\footnotetext{
${ }^{3}$ We allowed participants of any age to complete the task but excluded (without analysis) the 12 participants who reported ages over 55 . This was to discourage participants from misreporting their age to participate in the task.
} 
Before beginning the main set of 10 blocks, participants performed two practice blocks, each with only 3 items. These items were from categories not used during the main blocks. During these practice blocks, we provided feedback about both participants' old/new decisions (correct/incorrect) and their color memory (showing them what color they selected as well as what the actual color of the object had been).

\section{Results}

Participants successfully remembered which items from the category they had seen. The average hit rate was $77.7 \%$ (SEM: $\pm 0.9 \%$ ), and the average false alarm rate $23.4 \%$ (SEM: $\pm 1.0 \%$ ). Thus, participants were largely successful in distinguishing old from new items ( $d^{\prime}=1.65 ;$ SEM: \pm 0.04$)$. This accuracy level was higher than that in Experiment 1 ( $d^{\prime}$ difference: $\left.t(222)=2.92, p=0.004\right)$, as expected with one-fourth as many items stored in memory.

Looking only at hits (i.e., items participants had seen and reported they had seen), we analyzed the color report data as a function of participants' reported confidence. We binned the confidence into low (1-2), medium (3-4) and high (5-6) confidence to provide enough power. We found that participants were more accurate in reporting the color when they expressed higher confidence $(F(2,199)=41.43$, $p<0.0001$; see Figure 6A).

The Bayesian integration model predicts that greater certainty about the color of items should result in less bias toward the mean color of the category. Consistent with this prediction, we found that participants were less biased toward the center of the category when they expressed higher confidence $(F(2,199)=7.44, p=0.0007$; see Figure $6 \mathrm{~B})$. This finding is consistent with a model where participants' certainty about the color modulates how much they weight the gist information, as in the Bayesian integration model. In addition, the current data reveal that even at highest confidence levels, when participants have the lowest error, they still report a color that is biased toward the category center 
(mean bias at highest confidence: $18.0^{\circ} ; \mathrm{SEM}: 1.7^{\circ} ; p<0.001$ ). Thus, participants integrate the gist information with the item information even for items they remember, where they remember the color of the item and they report high confidence in this color memory.
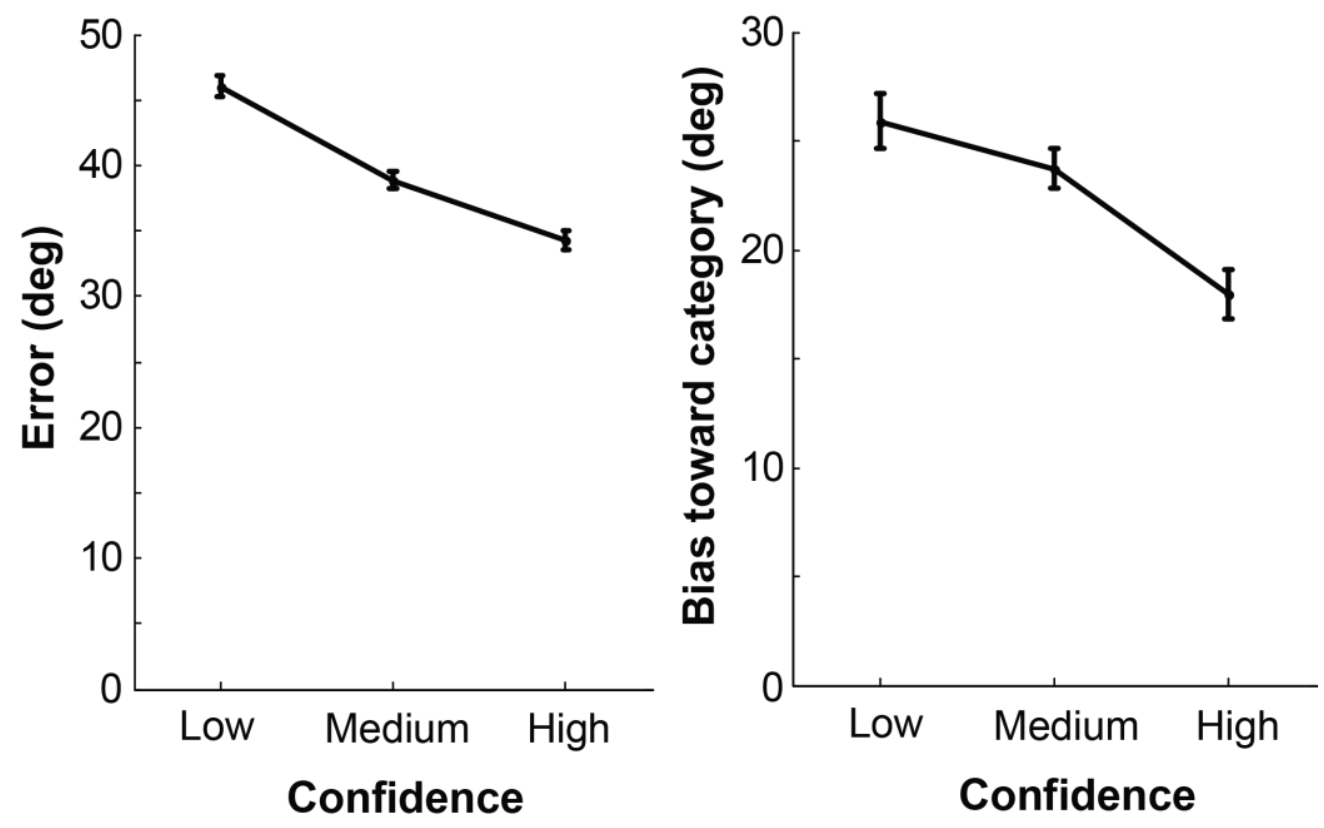

Figure 6. (A) Average error (in degrees) decreased as a function of participant's confidence. (B) As predicted by the Bayesian integration model, bias followed error, with the average bias also decreasing as error decreased and as participants reported greater certainty in the color they remembered for a particular item.

As in Experiment 1, we need to account for the possibility that the bias might arise because of a mixture of trials where participants report solely based on gist-only information and trials where they report based on solely on item information. This "mixture" account predicts that the color distribution consists of the gist-only distribution (calculated from color reports on false alarm trials), plus some remainder that represents item-specific information. Furthermore, the mixture account predicts that this remainder, representing item-specific information, is symmetric and is not biased toward the 
category's mean color (i.e., is centered around zero). These predictions are not consistent with the data at any confidence level.

In particular, no matter what proportion of hit trials we assume are actually gist-only response trials, the residual always shows a bias toward the mean color of the category at all confidence levels. As in Experiment 1, we consider all possible values of gist-only responses that are consistent with the data being a mix of gist-only responses plus item-based responses; in other words, we do not consider values where the appropriately weighted gist-only distribution is actually reliably greater $(p<0.05)$ than the old item color report distribution at any point, as this is inconsistent with the mixture account. As expected, this procedure leads to a maximum possible percentage of gist-only responses that decreases with higher confidence: At low confidence, the distribution is consistent with as many as $96 \%$ of responses being gist-only; at medium confidence levels, the distribution is consistent with as many as $56 \%$ of responses being gist-only; and high confidence, the distribution is consistent with as many as $16 \%$ of responses being gist-only. At all values, across all confidence levels, the distribution of responses is biased toward the category center even after removing a proportion of gist-only responses (i.e., the average response position is always toward the category center; see Figure 7; all $p^{\prime} s<0.05$ ). There is also a main effect of confidence-level on bias at all proportions of gist-only responses where multiple confidence levels can be assessed (e.g., 0-56\%; with all $\left.p^{\prime} s<0.05\right)$. 


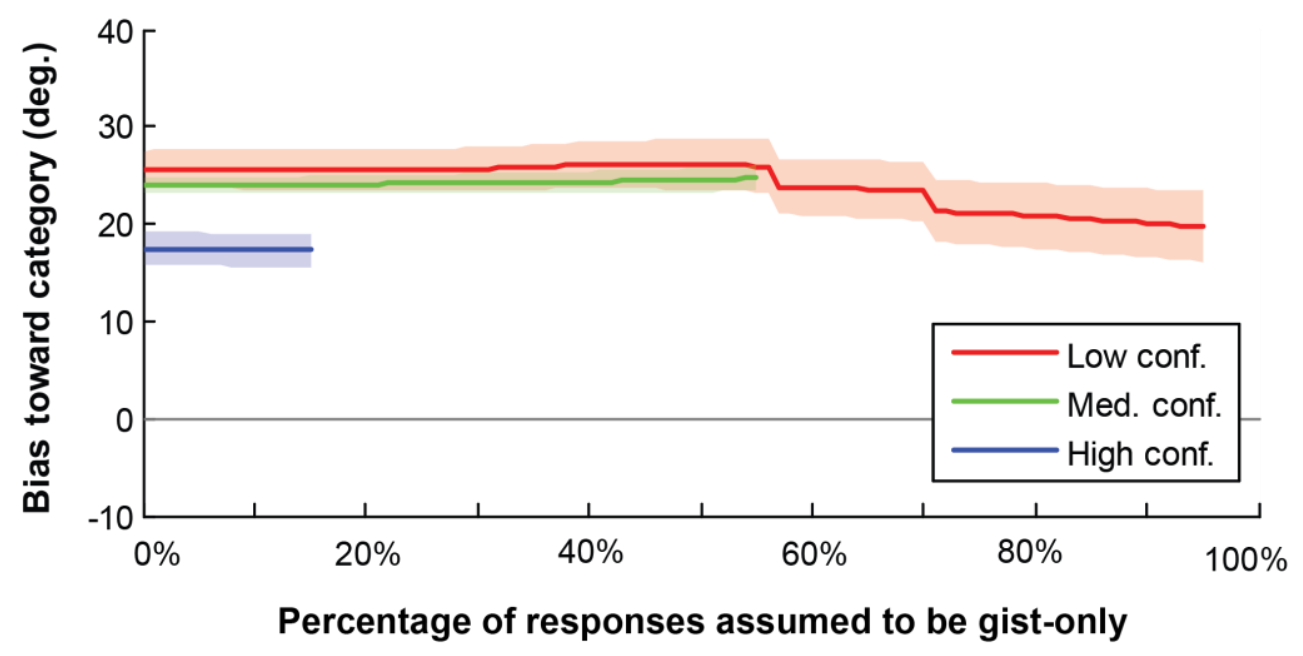

Figure 7. The remaining bias toward the category mean after removing the gist-only distribution. No matter what percentage of the responses are assumed to be gist-only responses ( $x$-axis), the itemspecific information in the residual continues to be biased toward the category's mean color ( $>0$ on the $y$-axis) at every confidence level. The shaded region represents the standard error of the mean across participants (note that this means it is not designed to compare the lines to each other, as this comparison is actually done within-subject; it is designed to show the lines differ from zero). The different color lines show the different levels of confidence that participants expressed in their color memory. Each of the lines extends only to the point where it becomes impossible to subtract more of the gist-only distribution without violating the assumption that the distribution is derived from a sum of both the gist-only distribution and item-specific responses (e.g., once the gist-only distribution is significantly greater than the item distribution at any point).

Thus, even if some proportion of participants' responses do reflect cases where participants had no item information and responded only based on category information, the remainder of the responses do not reflect unbiased reports of the color. Instead, participants appear to be systematically biased toward the mean color of the category even in the remaining trials where item-specific information was used. This pattern is observed across all confidence levels.

In summary, we find that participants are biased toward the category mean; that they are less biased when they are highly confident in their color reports, but nevertheless remain somewhat biased 
toward the category center even for highly confident trials; and that this bias is not purely a result of some responses reflecting only gist information.

\section{General Discussion}

Even when people remember having seen an item and remember the item's color, they nevertheless are systematically biased to report its color as closer to the mean color of its category than it was. This finding is consistent with the optimal Bayesian strategy: reporting a biased color reflects that participants used not only item-specific information but also category-level information to help them respond accurately, which results in less error on average than relying on only item-specific memories. This observation suggests that the gist information plays a role not just in false memories but also in true memories, i.e., accurate responding for remembered items. Furthermore, it suggests that memory distortion can have a clearly adaptive function: although participants' memories are biased, they are biased in a way that is consistent with minimizing their average error, as any bias toward the category center results in less error on average (see Appendix). Moreover, with greater uncertainty about the item's color (i.e., lower confidence in the color), participants show more bias toward the category mean, also consistent with the Bayesian integration framework's prediction.

Applying this Bayesian framework (Hemmer \& Persaud, 2014; Hemmer \& Steyvers, 2009b; Huttenlocher et al., 2000) to the case of gist-based false memories yields a novel perspective on the nature of false memories. In particular, in this Bayesian framework, false memories are part of a single continuum with true memories: for both false and true memories, people use information about the category to influence their responses. The more accurate their item-specific memory, the less biased they are by the category information ( $\sigma$ is small, bias is small); the less clear their item-specific memory, the greater their reliance on information about the category instead ( $\sigma$ is big, bias is big). In the case where participants possess no item-specific information at all ( $\sigma=$ infinity), they rely entirely on the 
category, giving rise to color reports that we think of as "false memory". Nevertheless, throughout the entire range from perfect memory to false memory, participants' responses can be thought of within the framework of a Bayesian system that is adaptively and optimally weighting the information available. This view is a significant departure from previous conceptualizations of the relationship between false memory and true memories, which have focused on dual-process models where people use the gist information only when item-specific information is unavailable (e.g., Brainerd \& Reyna, 2005). Instead, our data suggest that we should think about memory representations as hierarchical (Brady, Konkle \& Alvarez, 2011; Brady \& Alvarez, 2011), with gist information constraining and interacting with itemspecific information (i.e., acting as a prior on the item information in the Bayesian model). 


\section{False memory on a continuum with true memory}

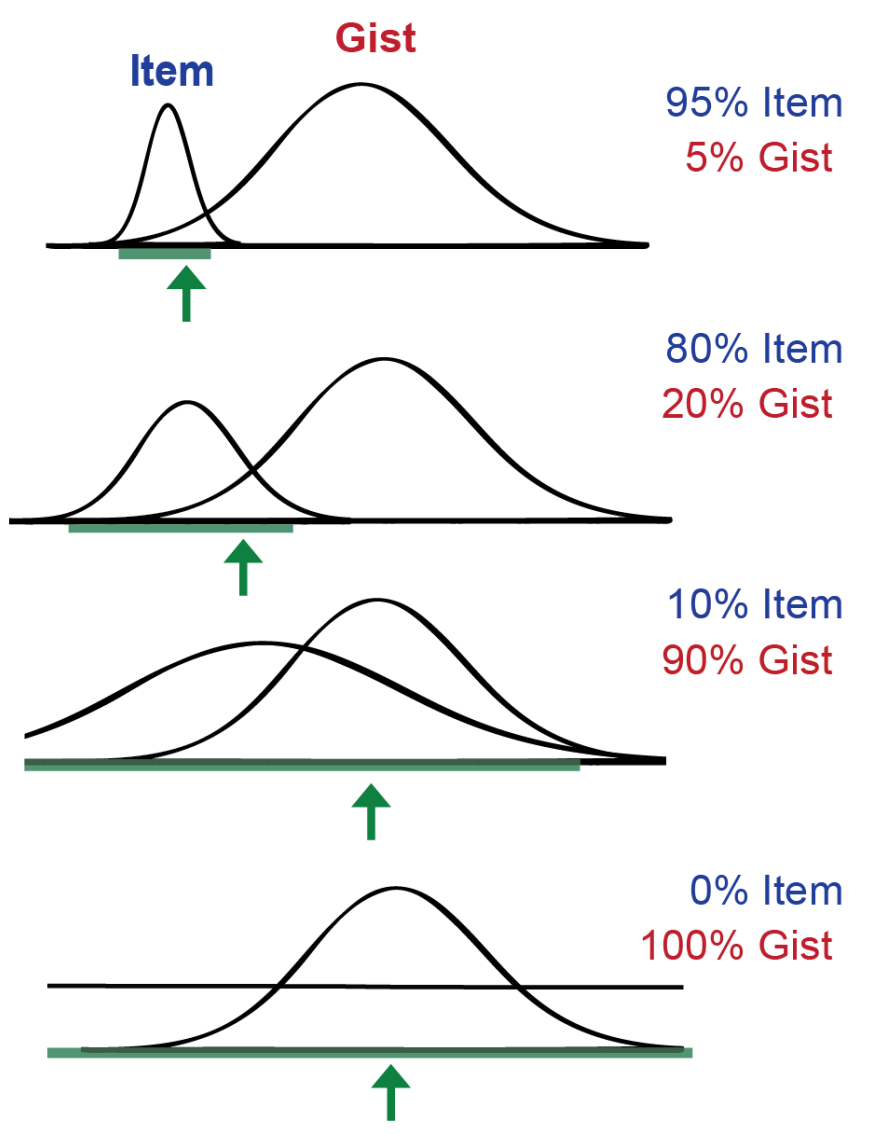

Figure 8. False memory on a continuum with true memory. When item memory is very precise, there is little bias towards the category-mean (the 'gist'). As item memory gets less and less precise, this bias increases. Ultimately, with no item information (a purely uniform distribution), participants rely entirely on gist ("false memory").

Continuous, analogue measures of long-term memory

The psychophysical method of adjustment allowed us to examine memory traces in detail. While previous work has used this method to examine the amount of detail present in long-term memory (Brady et al. 2013), the present study demonstrates that this method can provide deeper insights into the information that people store and represent by enabling us to examine visual long-term memory in an analog fashion. In particular, such analog methods allowed us to ask whether participants do indeed 
combine gist and item-specific information, whereas such a question is extremely difficult to ask when the verbatim trace is entirely sufficient to drive correct memory performance (as in most studies using old/new measures or using words as stimuli). Psychophysical methods that use continuous report for the actual stimulus value (as in the present study), or that use a continuous confidence measure (e.g., DeSoto \& Roediger, 2014), may also prove fruitful in other areas of the long-term memory literature where models have been difficult to distinguish (e.g., the all-or-none nature of recollection; Harlow \& Donaldson, 2013).

The adaptive nature of memory distortion

Memory distortion is traditionally thought of as a failure of our memory system. However, our results suggest that at least some distortion of true memories might instead be thought of as the outcome of a system that optimally weights all the information it has available. In particular, when people have both category-level gist information as well as item-specific information available, they appear to combine this information to arrive at an answer. This answer is biased, on average, thus yielding memory distortion. However, in this case, the distortion results from combining information from the gist with the item-specific information, leading people to be more accurate on average. Thus, in line with other emerging evidence (see Carpenter \& Schacter, 2017, 2018; Dewhurst, Anderson, Grace, \& van Esch, 2016; Howe, 2011; Schacter et al., 2011), it seems clear that memory distortion is not always maladaptive: in some cases, memory distortion can result from a memory system that optimally combines information from different kinds of memory traces in the service of the broader goals of the person.

\section{Acknowledgements}

This work was funded by a NSF CAREER BCS-0953730 to G.A.A, NIA AG008441 to D.L.S. and by NSF CAREER BCS- 1653457 to T.F.B. 


\section{References}

Anderson, J.R. \& Milson, R. (1989). Human memory: An adpative perspective. Psychological Review, 96, 703-719

Bar, M. (2004). Visual objects in context. Nature Reviews Neuroscience, 5(8), 617-629.

Bartlett, F. C. (1932). Remembering: An experimental and social study. Cambridge: Cambridge University.

Bjork, R.A. \& Bjork, EL. (1988). On the adpative aspects of retrieval failure in autobiographical memory. In M.M Gruneberg, P.E. Morris, \& R.N. Sykes, (Eds.), Practical aspects of memory: Current research and issues (Vol. 1, pp. 283-288). Chichester, Englad: Wiley).

Brady, T. F. and Alvarez, G.A. (2011). Hierarchical encoding in visual working memory: ensemble statistics bias memory for individual items. Psychological Science, 22(3), 384-392.

Brady, T. F., Konkle, T., Alvarez, G. A. and Oliva, A. (2008). Visual long-term memory has a massive storage capacity for object details. Proceedings of the National Academy of Sciences, USA, 105 (38), $14325-14329$.

Brady, T. F., Konkle, T.F., Gill, J., Oliva, A. and Alvarez, G.A. (2013). Visual long-term memory has the same limit on fidelity as visual working memory. Psychological Science, 24(6), 981-990.

Brady, T. F., Konkle, T, and Alvarez, G.A. (2011). A review of visual memory capacity: Beyond individual items and towards structured representations. Journal of Vision, 11(5):4, 1-34.

Brainard, D. H. (1997). The psychophysics toolbox. Spatial Vision, 10(4), 433-436.

Brainerd, C. J., \& Reyna, V. F. (1998). Fuzzy-trace theory and children's false memories. Journal of Experimental Child Psychology, 71(2), 81-129.

Brainerd, C. J., \& Reyna, V. F. (2002). Fuzzy-trace theory and false memory. Current Directions in Psychological Science, 11(5), 164-169. 
Brainerd, C. J., \& Reyna, V. F. (2005). The science of false memory. Oxford University Press.

Brainerd, C. J., Reyna, V. F., \& Mojardin, A. H. (1999). Conjoint recognition. Psychological Review, 106(1), 160.

Brewer, W. F., \& Treyens, J. C. (1981). Role of schemata in memory for places. Cognitive Psychology, 13(2), 207-230.

Carpenter, A.C., \& Schacter, D.L. (2017). Flexible retrieval: When true inferences produce false memories. Journal of Experimental Psychology: Learning, Memory, and Cognition, 43, 335-349.

Carpenter, A.C., \& Schacter, D.L. (2018). False memories, false preferences: Flexible retrieval mechanisms supporting successful inference bias novel decisions. Journal of Experimental Psychology: General. http://dx.doi.org/10.1037/xge0000391

Dewhurst, S.A. Anderson, R.J., Grace, L. and van Esch, L. (2016). Adaptive false memories: Imagining future scenarios increases false memories in the DRM paradigm. Memory \& Cognition, 44, 10761084.

Dewhurst, S. A., Thorley, C., Hammond, E. R., \& Ormerod, T. C. (2011). Convergent, but not divergent, thinking predicts susceptibility to associative memory illusions. Personality and Individual Differences, 51(1), 73-76.

Erdfelder, E., Auer, T. S., Hilbig, B. E., Aßfalg, A., Moshagen, M., \& Nadarevic, L. (2009). Multinomial processing tree models. Journal of Psychology, 217(3), 108-124.

Fisher, N. I. (1993). Statistical analysis of circular data. Cambridge University Press, Cambridge, UK. 
Guerin, S. A., Robbins, C. A., Gilmore, A. W., \& Schacter, D. L. (2012). Retrieval failure contributes to gistbased false recognition. Journal of Memory and Language, 66(1), 68-78.

Harlow, I. M., \& Donaldson, D. I. (2013). Source accuracy data reveal the thresholded nature of human episodic memory. Psychonomic Bulletin \& Review, 20(2), 318-325.

Hemmer, P. \& Steyvers, M. (2009). A Bayesian account of reconstructive memory. Topics in Cognitive Science, 1, 189-202.

Howe M.L. (2011). The adaptive nature of memory and its illusions. Current Directions in Psychological Science, 20, 312-315.

Huttenlocher, J., Hedges, L. V., \& Vevea, J. L. (2000). Why do categories affect stimulus judgment?. Journal of Experimental Psychology: General, 129(2), 220.

Johnson, M. K, Hashtroudi, S., \& Lindsay, D. S. (1993). Source monitoring. Psychological Bulletin, 114, 328

Koutstaal, W., \& Schacter, D. L. (1997). Gist-based false recognition of pictures in older and younger adults. Journal of Memory and Language, 37(4), 555-583.

Koutstaal, W., Verfaellie, M., \& Schacter, D.L. (2001). Recognizing identical vs. similar categorically related common objects: further evidence for degraded gist-representations in amnesia. Neuropsychology, 15, 268-289.

Lampinen, J. M., Copeland, S. M., \& Neuschatz, J. S. (2001). Recollections of things schematic: room schemas revisited. Journal of Experimental Psychology: Learning, Memory, and Cognition, 27(5), 1211.

Reyna, V. F., \& Brainerd, C. J. (1995). Fuzzy-trace theory: Some foundational issues. Learning and Individual Differences, 7, 145-162. 
Newman, E., \& Lindsay, D. S. (2009). False memories: What the hell are they for? Applied Cognitive Psychology, 23, 1105-1121.

Pelli, D. G. (1997). The VideoToolbox software for visual psychophysics: Transforming numbers into movies. Spatial Vision, 10(4), 437-442.

Schacter, D. L., Israel, L., \& Racine, C. (1999). Suppressing false recognition in younger and older adults: The distinctiveness heuristic. Journal of Memory and Language, 40(1), 1-24.

Schacter, D. L. (2001). The seven sins of memory. New York: Houghton Mifflin.

Schacter, D.L. (2012). Adaptive constructive processes and the future of memory. American Psychologist, 67, 603-613.

Schacter, D. L., Guerin, S. A., \& St Jacques, P. L. (2011). Memory distortion: an adaptive perspective. Trends in Cognitive Sciences, 15(10), 467-474.

Zhang, W., \& Luck, S. J. (2008). Discrete fixed-resolution representations in visual working memory. Nature, 453(7192), 233-235. 


\section{APPENDIX}

\section{Shifting responses toward the category center minimizes error}

In general, reporting a value that is shifted toward the category mean from the actual encoded memory trace will decrease expected error in situations where similar items are shown in similar colors, such as in the current experiment (see also Huttenlocher, Hedges \& Vevea, 2000; Brady \& Alvarez, 2011). This effect makes intuitive sense: If a participant knows something about what the items in a task were like in general (e.g., there were many green boats), then this gist information can inform the individual's specific item memories (e.g., the studied boat was probably on the greener side, even if the participant only remembers specifically that it was a blue-green).

The optimal amount of shift toward the category is represented by the equation given in the main text if the distribution of items from the category is normally distributed (as it is here) and a noisy memory trace is also normally distributed (Gelman, Carlin, Stern \& Rubin, 2003). In this equation, $r$ indicates the final response, $x$ indicates the noisy item memory ( $\sigma$ indicating the perceptual and memory noise, e.g., the standard deviation), and $\mu$ indicates the category mean ( $\tau$ indicating its standard deviation, e.g., how spread out items in the category are):

$$
r=\frac{\tau^{2}}{\sigma^{2}+\tau^{2}} x+\frac{\sigma^{2}}{\sigma^{2}+\tau^{2}} \mu
$$

This is the general form of the posterior for a hierarchical Bayesian model with a normal distribution as the prior and a normal distribution as the likelihood (Gelman et al. 2003). It takes the form of a weight $(\lambda)$ on the encoded memory trace $x$ and the remaining weight (1- $\lambda)$ on the category center:

$$
r=(\lambda) x+(1-\lambda) \mu
$$


Because of the way variance scales $\left(\operatorname{var}\left(a^{*} B\right)=a^{2} \operatorname{var}(B)\right)$, the error, on average, that participants would have if they derived their responses from this category-adjusted estimate $(r)$ is described in terms of mean squared error as:

$$
\operatorname{MSE}(r)=(\lambda)^{2} \sigma^{2}+(\lambda-1)^{2} \tau^{2}
$$

This compares to the average error of $\sigma^{2}$ participants could expect using just the encoded value without using the category information at all, e.g., $\operatorname{MSE}(x)$. We can then ask for what values of $\lambda \operatorname{MSE}(r)$ is less than $\operatorname{MSE}(x)$, e.g.:

$$
(\lambda)^{2} \sigma^{2}+(\lambda-1)^{2} \tau^{2}<\sigma^{2}
$$

Solving for $\lambda$ shows that $\operatorname{MSE}(r)$ is less than $\operatorname{MSE}(x)$ for values of $\lambda$ such that:

$$
\frac{\tau^{2}-\sigma^{2}}{\tau^{2}+\sigma^{2}}<\lambda<1
$$

It is easy to see that for all values of $\sigma^{2}>0$ - memory traces of individual items that have any memory or perceptual uncertainty at all - weighting the category mean will result in a smaller mean square error than not weighting the category information at all (e.g., $\frac{\tau^{2}-\sigma^{2}}{\tau^{2}+\sigma^{2}}$ will be less than 1 , indicating there is a value of $\lambda$ where $\operatorname{MSE}(r)$ is less than $\operatorname{MSE}(x))$. Since we know that participants' color perception and color memory are not $100 \%$ perfect, bias toward the category mean in the current experiment generally represents participants performing better than would be expected if they did not show such a bias.

In addition to this wide range of $\lambda$ values that result in less error than simply reporting the raw memory trace $(x)$, we can also compute the optimal value of $\lambda$ to minimize $\operatorname{MSE}(r)$. As specified above and in the main text, this is given, via Bayes formula, as: 


$$
\lambda=\frac{\tau^{2}}{\sigma^{2}+\tau^{2}}
$$

In other words, the noisier the memory trace - and the less spread out the items within the category are - the more people should weight the category mean in their responses to achieve minimal error.

\section{References}

Brady, T. F. and Alvarez, G.A. (2011). Hierarchical encoding in visual working memory: ensemble statistics bias memory for individual items. Psychological Science, 22(3), 384-392.

Gelman, A., Carlin, J. B., Stern, H. S., and Rubin, D. B. (2003). Bayesian Data Analysis. London: Chapman and Hall.

Huttenlocher, J., Hedges, L. V., \& Vevea, J. L. (2000). Why do categories affect stimulus judgment?. Journal of Experimental Psychology: General, 129(2), 220. 